\title{
A Preliminary Study of Relation Induction between HTML Tag Set and User Experience
}

\author{
Azusa Nakano ${ }^{1}$, Asato Tanaka ${ }^{1}$, and Masanori Akiyoshi ${ }^{2}$ \\ 1 Faculty of Applied Information Science, Hiroshima Institute of Technology, \\ 2-1-1 Miyake, Saeki-ku, Hiroshima, Hiroshima 731-5193, Japan \\ ${ }^{2}$ Faculty of Engineering, Kanagawa University, \\ 3-27-1 Rokkakubashi, Kanagawa-ku, Yokohama, Kanagawa 221-8686, Japan
}

\begin{abstract}
This paper addresses a preliminary study for relation identification between the HTML tag set and user experience. Today's Web technologies such as "HTML5" and "Ajax" enable content providers to design rich Web pages, sometimes complicated and not ease-of-use. On the other hand, "user experience" is getting more and more significant as everyone from young to elder people uses the Web. The design principle seems not to be established from "user experience" viewpoints, because it includes user practical activities. Therefore our approach is to collect user operations and user impressions as to the target Web pages, then induce relation between user impression and such collected data by mining technologies. This paper reports a preliminary experimental results towards such systematic analysis.
\end{abstract}

Keywords: Web interface, user experience, relation induction.

\section{Introduction}

This paper addresses a preliminary study for relation identification between the HTML tag set and user experience, which is considered to be difficult to identify simply by analyzing the collected user enquete as to Web design. Web design is drastically changing along with related technologies, for instance, "HTML5", "Ajax" and so forth. Moreover "user experience" includes a user's practical activities as indicated in the term, which seems to be slightly different from user impression. This means that the design guideline is complex so that it considers concrete user operations and evaluation.

We here address the systematic approach towards the Web design evaluation from "user experience" viewpoints. To achieve this goal, we introduce mining function approach to identify relation between the HTML tag set and user experience. As to "user experience", a user's operations such as "mouse move", "mouse scroll" and "mouse over" on the browser screen and "mouse click" on the hyper link are stored with time stamp, and his/her impression concerning the target Web pages is collected by enquete including several aspects of Web design and usability. If we could identify some sequential mouse operations as patterns, this may indicate specific features for "user experience". Also we focus

M. Kurosu (Ed.): Human-Computer Interaction, Part III, HCII 2014, LNCS 8512, pp. 49-56, 2014. (C) Springer International Publishing Switzerland 2014 
on enquete from subjective evaluation as to this specific features. Then relation identification will be systematically done by association rule mining between these two data set.

Based on the above-mentioned approach, this paper describes entire framework for this systematic identification and preliminary experimental results by using data collection system and executing a sequential pattern mining technique. Section 2 describes previous research on this field. Section 3 describes the framework for relation identification between the HTML tag set and user experience. Section 4 describes results on data collection and sequential pattern mining. Section 5 describes the findings and future work.

\section{Related Work}

Usability is argued from various viewpoints such as psychology, system functionality, user performance and so forth. The work in [1] reports the experimental results when executing task-based usability testing and guideline-base inspection as to the broad-reach Web portals. The paper tries to clarify the result of consistency along with the testing and inspection, using questionnaire-based data, but does not handle the concrete operational data concerning the target interface. From a user viewpoints to design the Web interface, the work in [2] discusses the interface from "semiotics" viewpoints and makes re-design of Web signs, which mainly handle the static design under "semiotics". This approach is slightly different from "user experience" aspect that we focus. The work in 3] directly handles "user experience" aspect when a user executes information search on the Web, which implements interactivity-oriented interfaces for the search. This indicates interactiveness provides effective and convenient way to a user, but limits to the keyword search task. The work in 44 discusses more closely as "user experience" from emotional aspects of Web design. Of course a user emotional is significant that affects "user experience", but mostly focuses on the guideline evaluation.

\section{Relation Induction Framework}

\subsection{Proposed Framework}

As mentioned in Section 1, our proposed relation induction framework utilizes user operational log and subjective evaluation on the Web interface. Fig. 1 indicates the overview of our proposed framework, which consists of "data collection system" and "data mining system".

The "data collection system" collects minute user operations for executing pre-defined task and enquete after accomplishing the task. The "data mining system" includes two mining subsystems; one is a sequential pattern mining and the other is an association rule mining.

The requirement of "data collection system" is to collect user operations on the Web page without being aware of it. So it should be embedded ordinary 


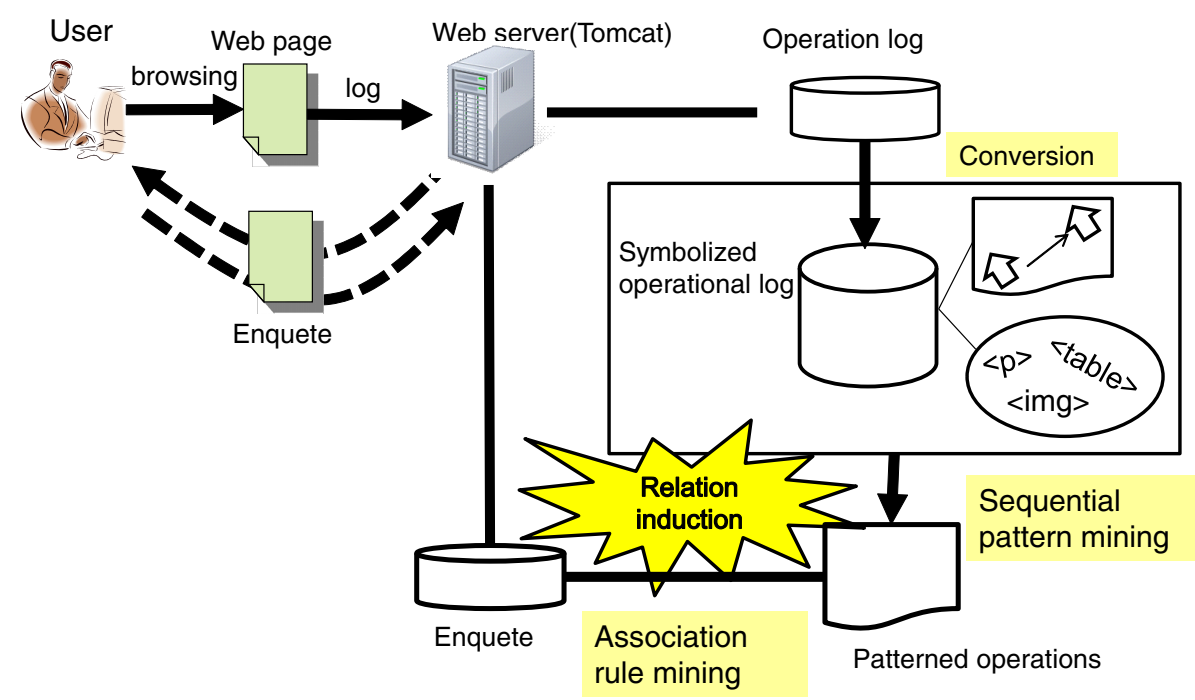

Fig. 1. Overview of Relation Induction Framework

Web browser to monitor and record the operations. Also the requirement of "data mining system" is to derive interpretative relationship between the HTML tag set and user evaluation. The HTML tag set is mostly categorized into two; one is structural tags and appearance tags. The former are tags for "itemization", "hyper link", "table" and so forth. The latter are tags for "colorization", "font", "layout" and so forth, which are basically recommended to use "〈span〉" in "HTML5". From capturing user operation viewpoints, the structural tags are seem to be significant, since those correspond to target components of user operations on the Web pages. Then our "data mining system" focuses on structural tags.

\subsection{Data Collection System}

Table 1 shows log items with time stamps; "mouse move" that are mouse x-y position, "mouse scroll" that is scrolling increment, "mouse click" that is operated on the hyper links, and "mouse over" on HTML tags that correspond to the Web components for the target task. Popular target tasks when a user uses Web pages are considered to be as follows; "information search" and "goods ordering". "Information search" means the situation that a user needs to know something and search information under browsing. "goods ordering" involves some procedures after deciding buying goods, for instance, input a user name and address, choose a payment method, designate the delivery time and so forth. Since we think the latter has less flexible operations than the former and the evaluation related to the former task indicates wide variety, we simply focus on the relation induction towards "information search" task. 
Table 1. Log Items using Mouse Event

\begin{tabular}{|l|l|}
\hline Type of mouse event & \multicolumn{1}{c|}{ Collected data } \\
\hline \hline mouse move & x position and y position \\
\hline mouse scroll & vertical increment \\
\hline mouse click & target url \\
\hline mouse over & target tag in the HTML source \\
\hline
\end{tabular}

The Web browser strictly limits the local access to storage resources such as HDD, so our system sends whole log data to the server by using Ajax technology. Fig. 2 shows the procedure of this data transferring, a part of Javascript code and collected data. To use Javascript code including Ajax, we need to modify the target HTML source for adding "〈script〉" tag. Therefore our system downloads the whole HTML related files at first and inserts the "〈script $\rangle$ " tag.

As indicated in Fig. 2, the actual collected log data using "!" as delimiter involves a user operations on one page as one line "string" data. Time stamps represent UTC.

\subsection{Data Mining System}

Characterizing Mouse Move. The time-series data of mouse events and transitions among Web pages are analyzed by using a sequential data mining technique, which induces a certain patterned user operation. To apply this mining, our system first converts relative mouse positions into absolute positions to identify mouse move such as "upward". Fig. 3 shows this conversion and the following fomula denotes this calculation where $y_{i}^{a b s}$ does absolute i-th y position, $y_{i}^{r e l}$ does relative $\mathrm{i}$-th y position, and $y_{k}^{s c r o l l}$ does $\mathrm{k}$-th vertical scroll increment.

$$
y_{i}^{a b s}=y_{i}^{r e l}+\sum_{k=1}^{i} y_{k}^{s c r o l l}
$$

Mouse move notation such as "upward" is necessary to extract patterns by sequential pattern mining. So we set eight directional mouse move notation as shown in Fig. 4. At this point, we do not discriminate the mouse move among the same directions, that is, ignoring the amount of pixel move, for instance, 10 pixel downward and 100 pixel downward are considered to be the same notation "downward". The symbolized mouse moves are given to numbers as indicated in Fig. 4 .

Extracting Mouse Patterns. The purpose of pattern mining is to extract a certain set of mouse operations embedded in when looking into one Web page and a certain set of transitions embedded in when traversing among several Web pages. The former is more significant because when searching information in the Web page, such specific operations may reflect users impression after finishing the browsing tasks. Of course the latter may also reflect users impression, especially whole evaluation-related impression. It is slightly easy to identify the latter, because a user actions towards links embedded in the Web page are mostly common under given tasks. Therefore this paper focuses on the former when users browse one Web page. 


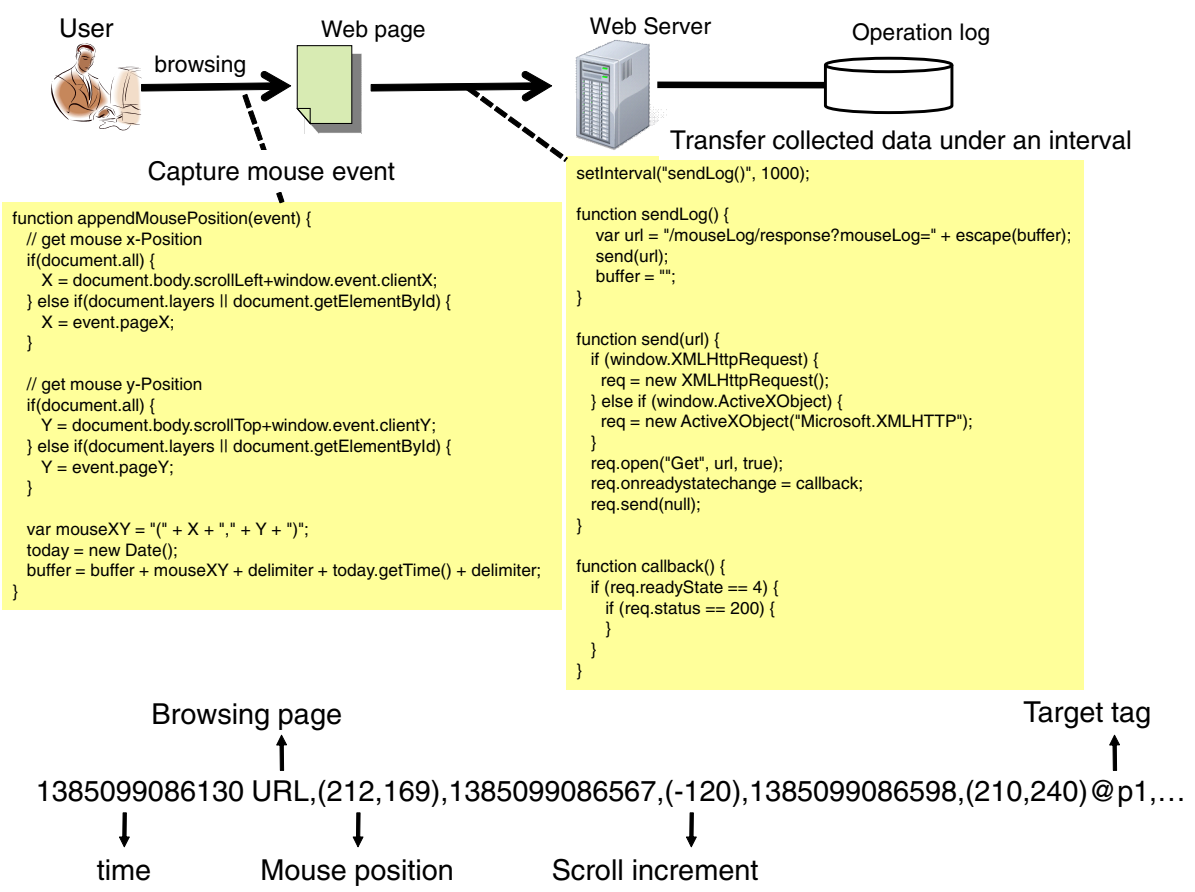

Fig. 2. Data Collection System Procedure

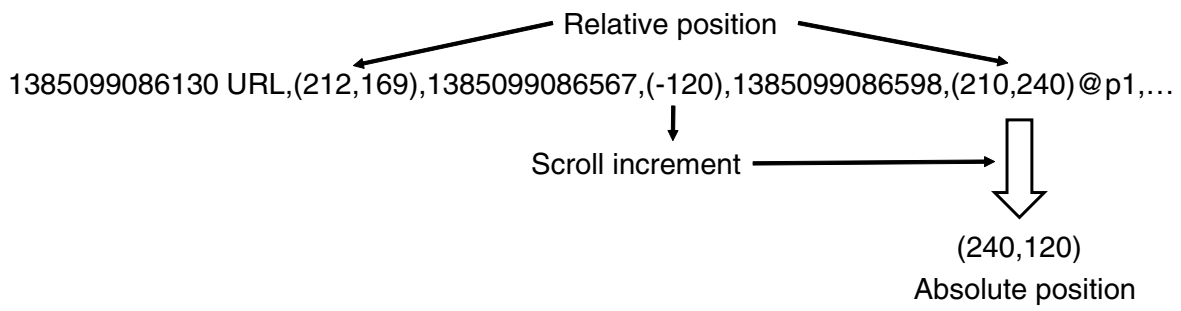

Fig. 3. Mouse Position Conversion

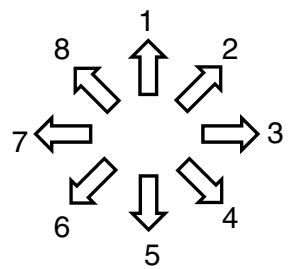

Fig. 4. Symbolized Mouse Move 
There exist several sequential pattern mining algorithms, and our requirement is to extract adjoining sequential patterns exactly, so the program proposed in 5] is used.

Identifying Relation. If some mouse sequential patterns are extracted from user operations, the next step is to identify relation between such specific user operations and user impression. Conceptually this approach is done by association rule mining, however practically it needs lots of mouse sequential patterns to execute such rule mining. In case of small set of mouse sequential patterns, other approach may be necessary.

\section{Experiment}

As preliminary study to identify relation using our proposed framework, this paper describes two experiments. One is to use three Web sites with three tasks for each Web site and collect impression after finishing each site. The other is to use one Web sites with three tasks for each Web site and collect impressions after each task. Figure 5 shows one sample screenshot of experimental Web pages; Guide for graduate school entrance examination, which is used in both experiments. As indicated in Figure 5, this Web page has " $\langle\mathrm{p}\rangle$ " HTML tag to depict some information. So after downloading this Web site entirely, target component IDs in the Web page are set.

Tasks set for this Web page are to grasp "[task1] difference between undergraduate school and graduate school", "[task2] subjects of entrance examination" and "[task3] how to study for entrance examination". The questions in enquete are as follows with "yes/no" response.

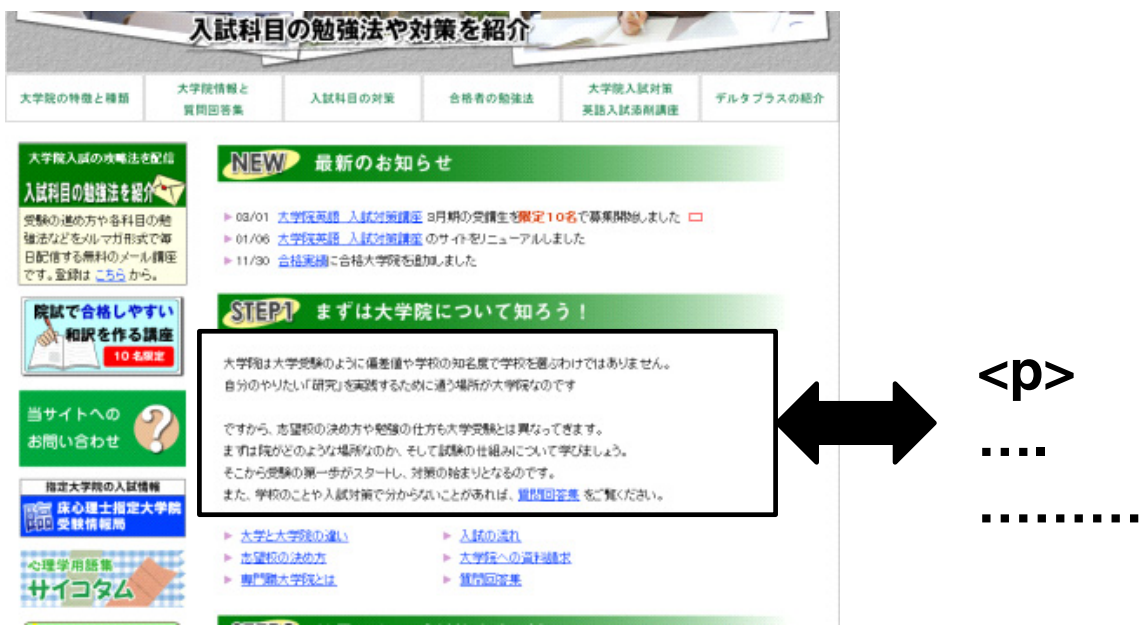

Fig. 5. Screenshot of Experimental Web Page 
Q1: Do you feel the unity concerning "color" and "design"?

Q2: Do you feel ease-of-use appearance concerning "menu", "character(font)", "graphics"?

Q3: Do you collect information you look for ?

Q4: What do you think is hard to understand in this Web page ?

Q5: Do you feel sense of intimacy?

Seven testers browse the three Web sites for the experiment 1, and nine testers do the one Web site for the experiment 2. The following are results on the common Web site "Guide for graduate school entrance examination" in both experiments

In the experiment 1 , there exists mouse move pattern " $\Rightarrow, \Uparrow, \Downarrow, \Uparrow "$. Table 2 shows the number of "yes" from the enquete.

Table 2. Question and response number

\begin{tabular}{|c|c|}
\hline \multicolumn{1}{|c|}{ question } & number of "yes" \\
\hline Q1: ... the unity $\ldots$ & 7 \\
\hline Q2: $\ldots$ ease-of-use appearance $\ldots$ & 6 \\
\hline Q3: $\ldots$ collect informaton $\ldots$ & 5 \\
\hline Q4: ... hard to understand $\ldots$ & 5 \\
\hline Q3: ... sens of intimacy $\ldots$ & 3 \\
\hline
\end{tabular}

In the experiment 2 , there exist mouse move patterns as indicated in Table 3 .

Table 3. Sequential patterns in each task

\begin{tabular}{|c|c|}
\hline task & mouse pattern \\
\hline \hline $\begin{array}{c}\text { difference between undergraduate school } \\
\text { and graduate school }\end{array}$ & $\Downarrow, \Rightarrow, \Downarrow$ \\
\hline subjects of entarance examination & $\Rightarrow, \Downarrow, \Rightarrow$ \\
\hline how to study for entrance examination & $\Rightarrow, \Uparrow, \Downarrow$ \\
\hline
\end{tabular}

Table 4 shows the number of "yes" from the enquete.

Table 4. Question and response number in each task

\begin{tabular}{|c|c|c|c|}
\hline \multirow{2}{*}{ question } & \multicolumn{3}{|c|}{ number of “yes" } \\
\cline { 2 - 5 } & task1 & task2 & task3 \\
\hline \hline Q1: $\ldots$ the unity $\ldots$ & 9 & 8 & 8 \\
\hline Q2: ... ease-of-use appearance $\ldots$ & 7 & 7 & 7 \\
\hline Q3: ... collect informaton $\ldots$ & 4 & 0 & 2 \\
\hline Q4: $\ldots$ hard to understand $\ldots$ & 4 & 6 & 4 \\
\hline Q5: $\ldots$ sens of intimacy $\ldots$ & 1 & 1 & 1 \\
\hline
\end{tabular}


As indicated in the results of sequential pattern mining towards mouse operations, we can get simple mouse move pattern that cannot explicitly relate to a certain meaningful move operation. This is caused by one reason that symbolized mouse move notations are generated under ignoring amount of pixel move. For instance, "downward" notations differ between the displayed page a user can gaze at a glance and the scrolled page. Other reasons are still under investigation.

\section{Summary}

This paper report a preliminary study for induction of relation between HTML tag set and user experience, which uses a user concrete mouse operations by capturing mouse events and corresponding HTML tag of "mouse over". The experimental results using actual Web pages show the problems to be tackled for applying mining techniques. In addition to mouse operations, a user eye movement needs to be added to capture a user intentional operations.

\section{References}

1. Granic, A., Mitrovic, I., Marangunic, N.: Usability evaluation of web portals. In: 30th International Conference on Information Technology Interfaces(ITI 2008), pp. $427-432$ (2008)

2. Islam, M.N.: Towards Designing Users' Intuitive Web Interface. In: Sixth International Conference on Complex, Intelligent and Software Intensive Systems (CISIS), pp. $513-518$ (2012)

3. Jianquan, L., Hanxiong, C., Furuse, K., Ohbo, N.: Using an interactive interface to support Web search for improving user experience. In: First International Conference on the Applications of Digital Information and Web Technologies(ICADIWT 2008), pp. 210-215 (2008)

4. Hussin, S.N., Mohd Lokman, A.: Kansei website interface design: Practicality and accuracy of Kansei Web Design Guideline. In: International Conference on User Science and Engineering (i-USEr), pp. 30-35 (2011)

5. Hirate, Y., Yamana, H.: Generalized Sequential Pattern Mining with Item Intervals Academy Publisher. Journal of Computers(JCP) 1(3), 51-60 (2006) 